\title{
Resilience-oriented intentional islanding of reconfigurable distribution power systems
}

\author{
Mohammad Hossein OBOUDI ${ }^{\mathbf{1}}$, Mohammad MOHAMMADI ${ }^{\mathbf{1}}$, \\ Mohammad RASTEGAR ${ }^{1}$
}

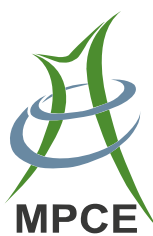

\begin{abstract}
Participation of distributed energy resources in the load restoration procedure, known as intentional islanding, can significantly improve the distribution system reliability. Distribution system reconfiguration can effectively alter islanding procedure and thus provide an opportunity to supply more demanded energy and reduce distribution system losses. In addition, high-impact events such as hurricanes and earthquake may complicate the procedure of load restoration, due to disconnection of the distribution system from the upstream grid or concurrent component outages. This paper presents a two-level method for intentional islanding of a reconfigurable distribution system, considering high impact events. In the first level, optimal islands are selected according to the graph model of the distribution system. In the second level, an optimal power flow (OPF) problem is solved to meet the operation constraints of the islands by reactive power control and demand side management. The proposed problem in the first level is solved by a combination of depth first search and particle swarm optimization methods. The OPF problem in the second level is solved in
\end{abstract}

CrossCheck date: 18 June 2019

Received: 1 January 2019/Accepted: 18 June 2019/Published online: 11 July 2019

(C) The Author(s) 2019

$\triangle$ Mohammad Hossein OBOUDI

mh.oboudi@shirazu.ac.ir

Mohammad MOHAMMADI

m.mohammadi@shirazu.ac.ir

Mohammad RASTEGAR

mohammadrastegar@shirazu.ac.ir

1 Department of Power and Control Engineering, School of Electrical and Computer Engineering, Shiraz University, Shiraz, Iran
DIgSILENT software. The proposed method is implemented in the IEEE 69-bus test system, and the results show the validity and effectiveness of the proposed algorithm.

Keywords Intentional islanding, Active distribution system, Distributed energy resources, Distribution system reconfiguration, Distribution system resilience

\section{Introduction}

The distribution system is the most vulnerable part of the power system, due to distributed structure, and low level of monitoring, controllability, and protections [1-3]. Hence, studies on effective methods for load restoration to improve the reliability of distribution systems have recently attracted more attention from scholars [1-5]. The load restoration has been considered as a network reconfiguration problem in the conventional distribution systems. Accordingly, once a fault occurs, the faulty section is isolated, and the tie-switches are closed to restore the outof-service customers [6, 7]. However, in the case of disconnection of a distribution system and upstream grid, this restoration method does not work [4].

The development of smart automation devices, such as digital protective devices, automatic feeder switches, and advanced metering infrastructure (AMI), motivates electric distribution utilities to modernize the grid [8-10]. In addition, the increasing penetration of distributed energy resources (DERs) in the distribution systems has changed the face of distribution systems from passive to active distribution systems (ADSs). Modern ADS can provide new opportunities to restore critical loads and to reduce the 
outage time, which enhances the reliability of the power system [8-13].

IEEE 1547 recommendation [14] encourages ADS to sectionalize the system into multiple networks, by participating DERs in the case of fault occurrence, which can improve load restoration. Accordingly, intentional islanding has been recently proposed and studied in [15-20] to improve the reliability of distribution systems. Intentional islanding is the procedure of selecting self-sufficient areas, called islands to restore critical loads through local DERs after a fault occurrence [15-20]. Feasible intentional islanding is characterized as high-priority load restoration, minimization of the number of switching operations, meeting the operation constraints, and maintaining the radial structure of islands [4]. Intentional islanding problem is a complex problem. Therefore, a decomposition strategy is usually used to split the problem into two sequential subproblems [16-20]. In the first sub-problem, primary islands are selected by determining the on/off status of switches in each line of ADS, by solving a mixed integer linear programming (MILP). For example, in [16], graph theory is used to model the ADS structure, and the branch and bound algorithm is applied to select primary islands. In [17] and [18], shuffled frog leap algorithm and particle swarm optimization (PSO) are respectively used to determine optimal primary islands. The authors of [19] employ a species-based quantum PSO (SQPSO) algorithm to obtain the primary islands. In the second sub-problem, various optimal decisions are made to meet the operation constraints of the primary islands. For example, [19] solves an optimal power flow (OPF) to meet the operation constraints. In addition, in [20], the demand side management (DSM) is proposed to meet the operation constraints of the islands in the second level.

In the mentioned literature [16-18, 20-25], power system reliability is guaranteed via the well-known $N-1$ contingency analysis. However, low-frequency high-impact events such as natural disaster may surpass the traditional consideration of reliability. For instance, hurricane Sandy, in 2012, caused concurrent 90 contingencies in the New York power distribution system [26]. This led to an economic loss of about $\$ 52$ billion [3]. In this regard, the resilience concept is introduced to the power systems. IEEE task force [27] defines resilience as "the ability to withstand and reduce the magnitude or duration of disruptive events, which includes the capability to anticipate, absorb, adapt to, or rapidly recover from such an event". Power system resilience can be classified into long-term and short-term analyses [28]. The long-term resilience refers to the flexibility of a critical network to changing conditions and new threats. The short-term resilience denotes the preventive and corrective measures carried out before, during, and after the natural disasters [28]. The resilience performance of a power system can be presented by a multi-phase resilience trapezoid curve, as shown in Fig. 1. This performance curve consists of 5 main sections: pre-disturbance resilient state, disturbance progress state, post-disturbance degraded state, restorative state, postrestoration state. The load restoration of ADS after extreme events can be considered in the context of the restorative state of the power system resilience. Accordingly, the predefined intentional islanding can be proposed as a prospective approach to increase the power system shortterm resilience. For example, in [3, 8], a MILP problem is solved to determine the primary islanding after concurrent outages of various lines caused by an adverse weather event. However, the operation constraints of islands are not considered in these works to check if the islanding is possible in a real case.

In this paper, a two-level approach is proposed to maximize the load restoration by intentional islanding of a reconfigurable distribution system. In the first level, primary islands are determined considering high-impact lowfrequency events, which may cause more than one contingency. Unlike previous works, the presence of tieswitches between different branches is also considered in the selection of primary islands. This may lead to meshed islands, which are not operationally accepted [4]. A modified MILP-based method is proposed to guarantee the radial structure of obtained islands in the first level. This is a kind of tree knapsack problem (TKP), which, in line with the [16], can be solved by heuristic algorithms. However, due to the random generation of the initial population in heuristic methods, many non-feasible solutions are produced that imposes a high computation burden to the solving procedure. This may cause heuristic methods to fail to perform in real large-scale systems. In this paper, a search method known as depth first search (DFS) is utilized to remove unfeasible solutions and limit the search space for the application of PSO method. In the second level, an OPF problem is solved to minimize the power losses in the islands, which considers the load management capability based on the load priorities (LPs).

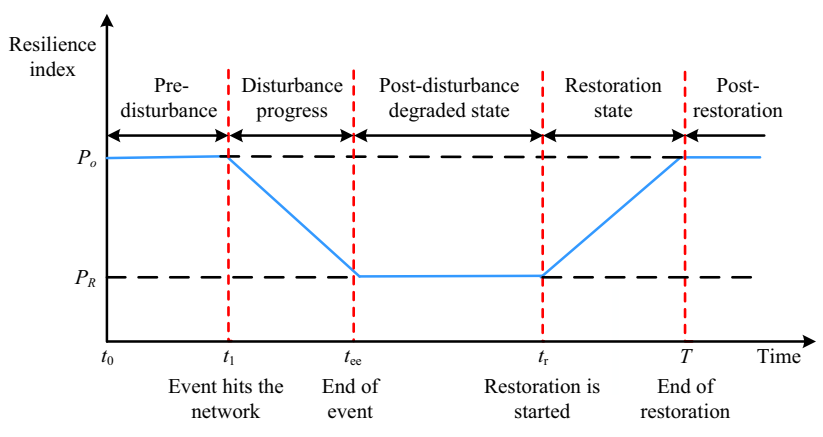

Fig. 1 A multi-phase resilience trapezoid performance curve [27] 
In conclusion, the main contributions of the paper are as follows: (1) proposing a restoration method to improve the resilience of ADS through the enhancement of the restorative state of the multi-phase resilience curve; (2) proposing a two-level intentional islanding method in a reconfigurable distribution system to maximize the load restoration and minimize the islands' energy losses; (3) introducing value of served energy (VOSE) to evaluate the resilience performance; (4) using the DFS method to limit the search space for the application of PSO method in the problem, which makes the application of the proposed method in large-scale systems possible.

The proposed method is examined in the IEEE 69-bus distribution system in different cases to show the effectiveness and validity of the proposed algorithm.

\section{Problem formulation}

\subsection{Problem description}

This section presents the two-level approach for intentional islanding in the case of fault occurrence in a distribution system. In the first level, the primary islands are obtained to maximize the value of load restoration. The presence of tie-lines is also considered in the island selection. The on/off status of the switches in the ADS lines is determined by solving an optimization problem, considering generation and demand balance and keeping the radial structure of the distribution system. It is worthwhile to mention that the radial structure of ADS may change to mesh structure in the case of switching the tie-lines on. Hence, the MILP proposed in [17] is modified, and considered as a TKP to find the radial primary island. The main reason for MILP application is the availability of various solvers that guarantee the convergence of the problem and are computationally effective [15, 29]. In the second level, an OPF is performed to minimize the power losses in primary islands, while operation constraints are also considered. The decision variables in the second level are the reactive power of DERs, the reactive power of reactive suppliers such as capacitor banks, and the active power of controllable loads. As a result, a feasible solution for the non-convex intentional islanding problem is found to restore high LPs and minimize power losses.

A graph model is used to represent the topological configuration of ADS such that, each node represents a load or a DER connected to the ADS through a bus. An "edge", numbered sequentially, represents the line connecting two nodes. The number of an edge is the number of the node with the higher number. The switch devices are assumed to be available to control all the lines. Generally, a conventional distribution system configuration is a radial configuration whereas an ADS with tie-lines can have a loop configuration in fault conditions. Hence, it is possible that more than one edge would be connected to one node. As an instance, an 11-bus distribution system and the related graph model are represented in Fig. 2.

In the following, the detailed mathematical formulations of the first and second levels are presented.

\subsection{First level: primary islanding}

Here, the VOSE is considered to select critical loads for restoration. The VOSE is the imposed cost to the customer in the case of an interruption in the electricity services [30]. VOSE usually depends on the customer types, i.e., residential, industrial or commercial, and the characteristics of interruption such as the duration and frequency of interruption. In this paper, the customers are prioritized based on the interruption cost presented in [31]. Accordingly, the customers are classified into three main categories: (1) residential customers with low LP; (2) small commercial and industrial customers with medium LP; (3) large commercial and critical customers with high LP [31]. Thus, in intentional islanding problem, VOSE for node $n$ is calculated as follows:

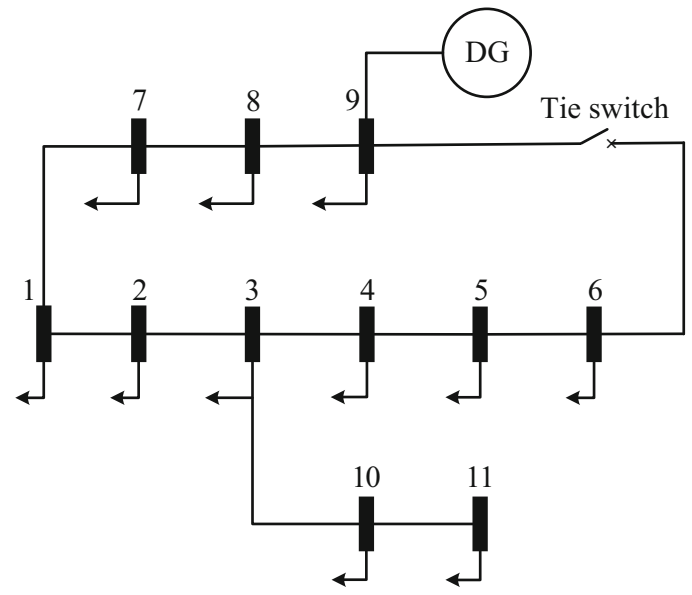

(a) Structure of the 11-bus ADS

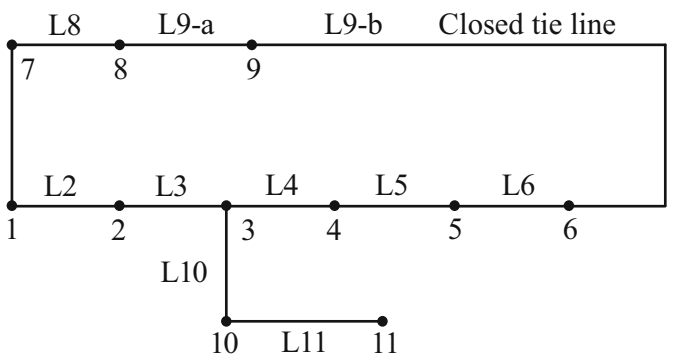

(b) Graph model of the 11-bus ADS

Fig. 2 Structure of 11-bus ADS and associated graph model 
$\operatorname{VOSE}_{n}=P_{n} t_{n} \times L P_{n}$

where $P_{n}, t_{n}$, and $L P_{n}$ are the active power, interruption duration, and amount of LP at node $n$, respectively. The main idea in the first level is to select the loads with the highest VOSE for restoration by DER units. Hence, the objective function would be:

$\max \left(\sum_{n \in N} \operatorname{VOSE}_{n} \times \mu_{n}\right)$

where $\mu_{n}$ is the binary variable indicating the serving status of the node $n$, it is 1 when the node $n$ is served, otherwise, it is 0 and $N$ is the set of nodes. Following constraints should be met in each island:

$\sum_{i \in I} P_{i} \leq \sum_{d \in D} P_{d}^{g} \quad \forall D \subset I$

$\mu_{d}=1 \quad \forall d \in D$

$\sum_{(a, b) \in I} \lambda(a, b)=\sum_{i \in I} \mu_{i}-1$

where $d$ is the index of nodes connected to DERs; $D$ is the set of nodes connected to DERs in the island; $i$ is the index of nodes in the island; $I$ is the set of selected nodes in the island; $P_{i}$ is the active power consumption in the island; $P_{d}^{g}$ is the active power generated by connected DER to node $d$ in the island; and $\lambda(a, b)$ is a binary variable, indicating the edge status of two adjacent nodes $a$ and $b$, selected in the primary island. Regarding the graph model, when an edge for connecting nodes $a$ and $b$ is available, $\lambda(a, b)$ is 1 ; otherwise, it is 0 .

In (3), it is verified that the generation of DERs in an island should be more than the load consumption. In (4), the node connected to DER is selected as the root node, since DER supplies the selected loads. Therefore, the nodes connected to DERs should be available in the island. In (5), the radial structure of islands is verified so that a set of nodes in an island forms a unique path. In other words, in a radial structure, the graph connecting all nodes has a unique path between two nodes, and the number of the edges is equal to that of nodes minus one. It is worth mentioning that the presence of a tie-line may change the ADS structure from a radial to loop structure. Therefore, (5) is the proposed modification on the conventional MILP methods to verify the radial structure of the islands.

It is shown in [32] that for a graph containing $n$ nodes, there are $2^{n-1}$ possible islands, although, many of them are not acceptable because of failing to satisfy (3). Reducing the unacceptable solutions to increase computational speed plays a vital role in the application of such methods. To this end, a limited search method known as DFS is performed in this paper to find all possible islands, satisfying (3). DFS is an algorithm to search a graph data structures. Here, the root node to search an island is the node connected to DER. The search is continuing, node by node, until (3) is met. Detailed mathematical modeling of this method can be found in [32].

In this paper, a heuristic methodology is used to solve the optimization problem (2)-(5) by PSO. The flowchart of the proposed methodology is presented in Fig. 3. As shown in this figure, for each DER, DFS is performed. $k$ is the index of DER and $M$ is the number of DERs. The possible islands determined by DFS are the candidates for primary island formation in the PSO problem. These islands are considered as particles in PSO in the form of input vectors to solve the optimization problem in (2)-(5). In details, for every particle vector, a binary decision variable is defined, and the results are obtained in a hierarchical procedure. First, the objective function (2) is calculated; then, the active power balance constraint in (3) is checked; and finally, the radial connectivity constraint in (5) is verified. It is worth mentioning that increasing the penetration levels

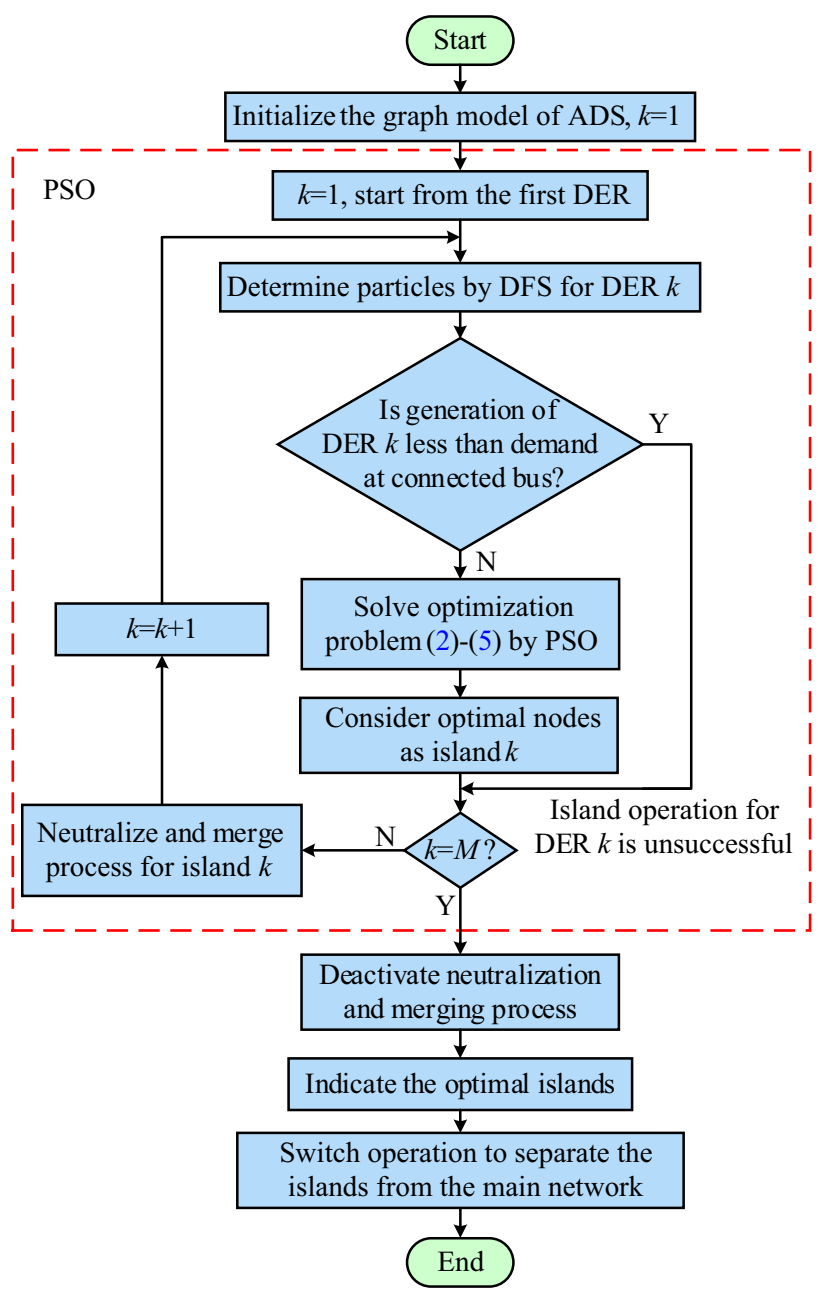

Fig. 3 Procedure of primary island selection 
of DERs in ADS may cause some nodes to be in more than one island. The solution is to combine these islands to obtain one island. To this end, when a primary island is obtained, the VOSE and the active power of the selected nodes are set to be zero for the evaluation of other islands. This process is named neutralizing, which can lead to a larger island by combining islands. In addition, the selected island is merged to reduce the search space for the other islands. When all primary islands are obtained, the neutralizing procedure is deactivated, and the main load and VOSE of nodes are reassigned. Finally, the optimal islands are determined. In order to separate the selected island from other sections, only the boundary lines, which connect the island to the neighboring nodes, are disconnected by the operation of switches. The number of the switch operation depends on the network structure. The procedure of the primary island selection is presented in Fig. 3.

\subsection{Second level: OPF}

The primary islands should be checked for a feasible operation. The island is likely adjusted to satisfy the operation constraints. To this purpose, optimal load management is considered through OPF calculations. Total power losses in each island are considered as the objective function, subject to voltage limitations, lines capacity constraints, and active and reactive power generation restriction. The mathematical model of the proposed OPF is represented as follows:

$\min P_{\text {loss }}^{j} \forall j \in J$

s. t.

$P_{\text {loss }}^{j}=\sum_{(a, b) \in I_{j}} G_{a b}\left(U_{a}^{2}+U_{b}^{2}-2 U_{a} U_{b} \cos \phi_{a b}\right)$

$Q_{l o s s}^{j}=\sum_{(a, b) \in I_{j}} B_{a b}\left(U_{a}^{2}+U_{b}^{2}-2 U_{a} U_{b} \cos \phi_{a b}\right)$

$P_{n}=\sum_{(a, b) \in I_{j}}\left|U_{a} U_{b} Y_{a b}\right| \cos \left(\delta_{a}-\delta_{b}+\phi_{a b}\right) \quad \forall n \in I_{j}$

$Q_{n}=\sum_{(a, b) \in I_{j}}\left|U_{a} U_{b} Y_{a b}\right| \sin \left(\delta_{a}-\delta_{b}+\phi_{a b}\right) \quad \forall n \in I_{j}$

$\sum_{n \in I_{j}} P_{n}+P_{\text {loss }}^{j} \leq \sum_{d \in I_{j}} P_{d}^{g}$

$\sum_{n \in I_{j}} Q_{n}+Q_{\text {loss }}^{j} \leq \sum_{d \in I_{j}} Q_{d}^{g}$

$U_{n, \min } \leq U_{n} \leq U_{n, \max } \quad \forall n \in I_{j}$

$-Q_{D E R, \max } \leq Q_{D E R} \leq Q_{D E R, \max }$

$0 \leq P_{D E R} \leq P_{D E R, \max }$
$0 \leq Q_{c} \leq Q_{c, \max }$

$S_{a, b} \leq S_{a, b, \max } \quad \forall a, b \in I_{j}$

where $P_{\text {loss }}^{j}$ and $Q_{\text {loss }}^{j}$ are the active and reactive power losses in island $j$, respectively; $J$ is the set of primary islands; $I_{j}$ is the set of selected nodes in the island $j ; U_{a}$ and $U_{b}$ are the voltage magnitudes of nodes $a$ and $b$, respectively; $\delta_{a}$ and $\delta_{b}$ are the phases of nodes $a$ and $b$, respectively; $Q_{d}^{g}$ is the reactive power generated by connected DER to node $d$ in the island; $\phi_{a b}, B_{a b}, G_{a b}$, and $Y_{a b}$ are the magnitude, real part, imaginary part, and the phase of admittance between nodes $a$ and $b$ in the primary island, respectively; $U_{n, \min }$ and $U_{n, \max }$ are the minimum and maximum voltage limits, respectively; $Q_{D E R \text {, max }}$ is the maximum reactive power injected by DER; $Q_{c, \max }$ and $S_{a, b, \max }$ are the maximum reactive power generated by capacitor banks, and the maximum capacity of the line connected between nodes $a$ and $b$, respectively; and $P_{D E R}$ and $P_{D E R, \max }$ are the active power and maximum active power injected by DER, respectively.

In (7) and (8), the active and reactive power losses in island $j$ are calculated. In (9) and (10), active and reactive power for node $n$ are obtained, respectively. In (11) and (12), the active and reactive power balance between generation and load consumption is verified. Voltage constraint for nodes is represented in (13). It is assumed that DERs can provide or consume reactive power in an acceptable range. This is mathematically presented in (14). In (15), active power generation of DERs is capped to their capacity $P_{D E R \text {, max }}$. In (16), the reactive power generation of capacitor banks is limited. Finally, the line capacity constraint is modelled in (17).

It is worthwhile to mention that direct load control is considered as the DSM program in this paper [33]. Hence, after a short notice, a part of participated demands can be curtailed in the case of a violation from each of the OPF constraints. Thus, when the operation constraints such as line capacity are violated, the controllable load is shed to meet the OPF constraints. Accordingly, the load is mathematically separated into controllable and uncontrollable parts as follows:

$\left\{\begin{array}{l}P_{n}=\left(\alpha_{n}+\beta_{n}\right) P_{n} \quad \forall n \in I_{j} \\ 0 \leq \alpha_{n} \leq 1 \\ 0 \leq \beta_{n} \leq 1\end{array}\right.$

where $\alpha_{n}$ and $\beta_{n}$ determine the share of controllable and uncontrollable demands in node $n$, respectively, $\alpha_{n}+\beta_{n}=1$. In this paper, the proposed OPF is performed by DIgSILENT power factory software [34]. 


\subsection{Application of proposed two-level method}

In the case of any disturbances in the operation of ADS, the proposed two-level method is applicable. If a fault occurrence in the system led to a disconnection from the main grid, the proposed two-level method would be implemented to achieve the optimal islanding. Sometimes, a fault separates some sections of ADS, while the ADS and upstream grid are still connected. In this case, conventional reconfiguration of the network is performing by closing the available tie-lines. Therefore, the isolated part may be connected to the network by the tie-lines. In this case, only an OPF problem is solved to improve the operation of the system, considering DER presence. It is also possible that after a fault occurrence and closing tie-lines, a part of the network remains isolated from the grid. In this case, if DERs are available in the separated parts, the proposed two-level method would be applicable in the isolated part to supply high priority loads. Abovementioned applications of the proposed two-level method are concluded in Fig. 4.

\subsection{Resilience metrics}

Resilience curve is used to quantify the resilience level by defining the performance loss (PL). PL is defined as the difference between the normal performance level and the resilience curves, which has a trapezoid shape, as presented in Fig. 1. PL index is usually represented by a number between 0 and 1 , where 0 and 1 are the best and the worst resilience indices, respectively. PL can be formulated by:

$P L=\frac{\int_{t_{1}}^{T} P_{o}(t) \mathrm{d} t-\int_{t_{1}}^{T} P_{R}(t) \mathrm{d} t}{\int_{t_{1}}^{T} P_{o}(t) \mathrm{d} t}$

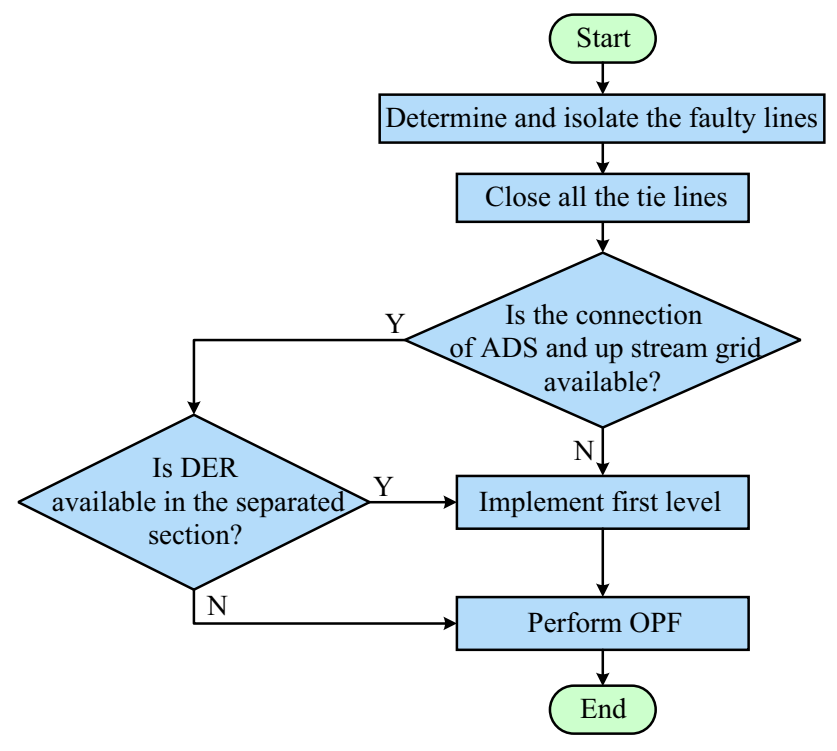

Fig. 4 Application procedure of proposed method where $P_{o}(t)$ and $P_{R}(t)$ are the values of the normal performance and resilience performance, respectively; $t_{1}$ and $T$ are the event hit time and the recovery end time, respectively. In this paper, VOSE is also proposed as the resilience performance index. The proposed islanding method in this paper has impacts on the restorative procedure in the resilience curve, and the resilience metrics are reported in the numerical studies.

\section{Numerical studies}

\subsection{Assumptions and scenario definition}

The well-known IEEE 69-bus distribution system is selected as the test system, as shown in Fig. 5. The rated current of tie-switches is $100 \mathrm{~A}$. The load demand of nodes is assumed to be the same as presented ones in [35]. Seven DERs are connected to ADS, and the capacities and locations are presented in Table 1, where the total capacity is $1055 \mathrm{~kW}$. The location of DERs is selected based on [16], and the generation capacity is considered around $30 \%$ of the total load, which is in line with the recommendations in [36]. The nominal voltage is $12.6 \mathrm{kV}$, and the maximum and minimum voltage limitations are, respectively, set to be $U_{\max }=1.05$ p.u. and $U_{\min }=0.95$ p.u..

DERs $1,3,4,5$, and 7 are small gas turbines as conventional resources that operate at unity power factor during the grid-connected mode. However, in the intentional islanding mode, the power factor can be set within the range of 0.8 lag and 0.8 lead. DER 2 and DER 6 are renewable resources, such as wind turbines, operating in unity power factor in both grid-connected and islanding conditions. The capacitor bank can supply the required reactive power in these points, providing reactive power in 10 steps. The nominal rating of these capacitor banks is considered to be $80 \%$ of the capacity of renewable generators. It is assumed that all ADS components operate in their nominal capacity. The load LPs and the amount of responsivity of loads are tabulated, respectively, in Tables 2 and 3. The outage duration of the substation connecting the upstream grid and ADS is assumed to be 24 hours, and the repair duration of the lines are considered to be 18 hours, as presented in [37]. The proposed method for primary island selection is provided in MATLAB software, and OPF is performed by DIgSILENT power factory software.

The proposed method is implemented in the test system, and the results are presented in this subsection. In order to demonstrate the performance of the proposed method for resilience studies, two scenarios are designed. In scenario 1 , it is assumed that the connection of ADS and the 


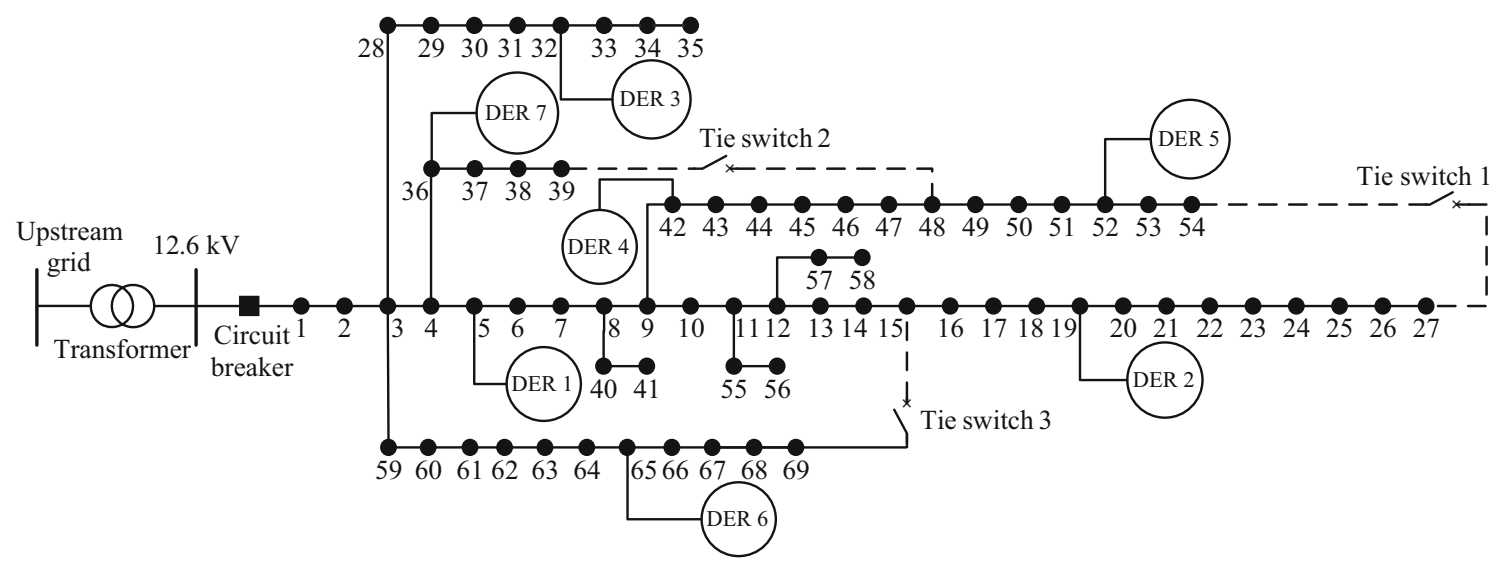

Fig. 5 Modified IEEE 69-bus test distribution system

Table 1 Characteristics of installed DERs

\begin{tabular}{lcc}
\hline DER number & Node number & Capacity $(\mathrm{kW})$ \\
\hline 1 & 5 & 35 \\
2 & 19 & 200 \\
3 & 32 & 40 \\
4 & 42 & 120 \\
5 & 52 & 400 \\
6 & 65 & 100 \\
7 & 36 & 160 \\
\hline
\end{tabular}

Table 2 Priority of loads [16]

\begin{tabular}{lrl}
\hline $\begin{array}{l}\text { Priority } \\
\text { level }\end{array}$ & $\begin{array}{l}\text { Priority }(\$ / \\
\mathrm{kWh})\end{array}$ & Node number \\
\hline 1 & 100 & $6,9,12,18,35,37,42,51,57,62$ \\
2 & 10 & $\begin{array}{l}\text { Other nodes } \\
3\end{array}$ \\
& 1 & $\begin{array}{l}7,10,11,13,16,22,28,38,43-48, \\
60,63\end{array}$ \\
\hline
\end{tabular}

Table 3 Load controllability of nodes

\begin{tabular}{llll}
\hline Node number & Load type & $\alpha$ & $\beta$ \\
\hline $24,26,27,34,39-41,43-44,48$, & $100 \%$ & 1.0 & 0 \\
$53-56,58,66-69$ & controllable & & \\
$11,13,16,21,38$ & $40 \%$ & 0.4 & 0.6 \\
& controllable & & \\
Other nodes & Uncontrollable & 0 & 1.0 \\
\hline
\end{tabular}

upstream grid is lost as a high impact event, and the twolevel method is implemented for load restoration. In scenario 2, it is assumed that more than one failure occur in the system due to a high impact event, while the distribution system and the upstream grid remain connected. These scenarios are supposed to be the consequences of high impact events. The proposed two-level load restoration method is implemented, and the results are reported in the following.

\subsection{Results of scenario 1}

In this scenario, line 1 is interrupted by a fault, and the connection of ADS and the upstream grid is disconnected. The proposed method is applied, and the feasible islands are concluded in Table 4.

At the first stage, the tie-switches are closed, and primary islands are determined. For example, as presented in Table 4, the particle vector obtained by DFS for DER 2 at node 19 are nodes $13-27$. Then, the modified MILP is solved by PSO, and nodes 18-22 are selected as the primary island 2. Two switches operate to separate the optimal island from other parts. Accordingly, the boundary lines connecting the nodes 17 and 18, and nodes 22 and 23 are opened. The effect of neutralization and merging can be found in island 7. Before selecting island 7, other islands are merged and neutralized. In island 7, the DFS selects nodes $7,10,29,36,37,43-47,59,60,69$ as particles, including the primary neutralized islands 1 and 4 . Then, by solving the optimization problem, nodes $7,36,37$ and neutralized islands 1,4 are selected as the optimal island. Then, the unmerging is performed and neutralization is deactivated. Finally, a larger island by combining islands 1, 4 , and 7 is obtained. It is interesting to see that closing tieswitch 1 has made the formation of island 5 possible. Switching tie-switch 1 on, the DFS has selected nodes 18-27 besides nodes $51-54$ as candidates. Finally, nodes 24-27 and 51-54 have been selected for the optimal formation of island 5. It is essential to mention that all DERs generate more than the selected island demand. Hence, it is possible that the maximum capacity of DERs is not utilized in the primary island formation. For example, in island 5, the generation capacity of DER 5 is $400 \mathrm{~kW}$, while the 
Table 4 Characteristics of islands with LPs

\begin{tabular}{|c|c|c|c|c|c|}
\hline Island & Candidate node determined by DFS & $\begin{array}{l}\text { Selected node determined by } \\
\text { PSO }\end{array}$ & $\begin{array}{l}\text { Restored load } \\
(\mathrm{kW})\end{array}$ & $\begin{array}{l}\text { VOSE }(\$ / \\
\text { hour })\end{array}$ & $\begin{array}{l}\text { Number of switch } \\
\text { operation }\end{array}$ \\
\hline 1 & $1-7,28,36,59$ & $1-6,28$ & 28.60 & 520 & l \\
\hline 2 & $13-27$ & $18-22$ & 180.30 & 7155 & 2 \\
\hline 3 & $29-35$ & $31-35$ & 39.50 & 935 & 1 \\
\hline 4 & $8-10,43-47$ & $8,9,42$ & 109.35 & 4185 & l \\
\hline 5 & $18-27,51-54$ & $24-27,51-54$ & 379.00 & 13780 & 3 \\
\hline 6 & $1-6,14-15,28-36,59-69$ & $61-68$ & 94.40 & 4630 & 2 \\
\hline 7 & $\begin{array}{l}\text { Neutralized islands } 1,4,6 \text {, nodes } 7,10,29,36,37 \text {, } \\
\quad 43-47,59,60,69 \text {, }\end{array}$ & $\begin{array}{l}\text { Neutralized islands } 1,4 \text {, nodes } \\
\quad 7,36,37,59\end{array}$ & 145.40 & 8564 & 6 \\
\hline Total & & & 976.55 & 39769 & 14 \\
\hline
\end{tabular}

Table 5 Results of running OPF in second stage

\begin{tabular}{llll}
\hline $\begin{array}{l}\text { Island } \\
\text { number }\end{array}$ & $\begin{array}{l}\text { Active power } \\
(\mathrm{kW})\end{array}$ & $\begin{array}{l}\text { Reactive power } \\
(\mathrm{kvar})\end{array}$ & $\begin{array}{l}\text { Power loss } \\
(\mathrm{kW})\end{array}$ \\
\hline $1,4,7$ & 282.6 & 238.0 & 0.5 \\
2 & 182.6 & 119.7 & 0.1 \\
3 & 39.6 & 28.0 & 0.1 \\
5 & 379.0 & 275.5 & 1.0 \\
6 & 94.5 & 65.6 & 0.1 \\
\hline
\end{tabular}

demand is $379 \mathrm{~kW}$. Thus, $21 \mathrm{~kW}$ of generation is curtailed in this island. The results presented in Table 4 also show that the total restored load and VOSE are $976.55 \mathrm{~kW}$ and 39769 \$/hour, respectively.

In the second stage, the operation of primary islands is optimized by controlling the reactive power generation of resources and load curtailment. The results are presented in Table 5. The results show that DERs and capacitor banks provided sufficient reactive power to converge the OPF solution. The total power loss is $0.17 \%$ of the total generation, which is considerably low. The main reasons for this are the separation of the network into small islands, which leads to the short distance between the generation and loads. The obtained feasible islands are presented in Fig. 6 .

For the sake of comparison, the result of implementing the proposed intentional islanding procedure without considering the tie-lines are presented in Fig. 7. These results are in line with the presented results in [17, 20]. It should be mentioned that without tie-lines, the total restored load is $920.5 \mathrm{~kW}$, which is $6 \%$ less than the case with tie-lines. In addition, total VOSE in the case of without tie-lines is 32049 \$/hour, which is $11.8 \%$ less than that of with tielines.

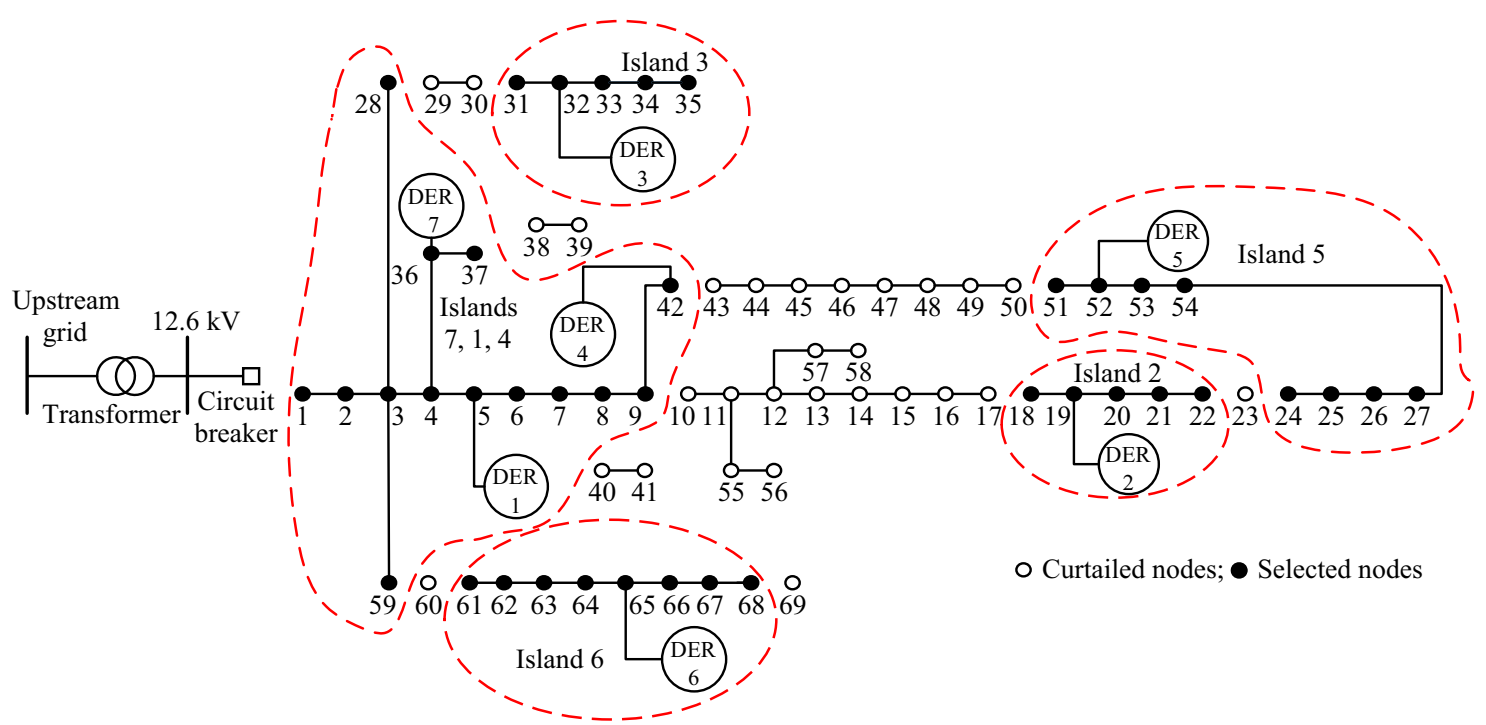

Fig. 6 Islanding formation by applying the proposed method in scenario 1 with LPs 


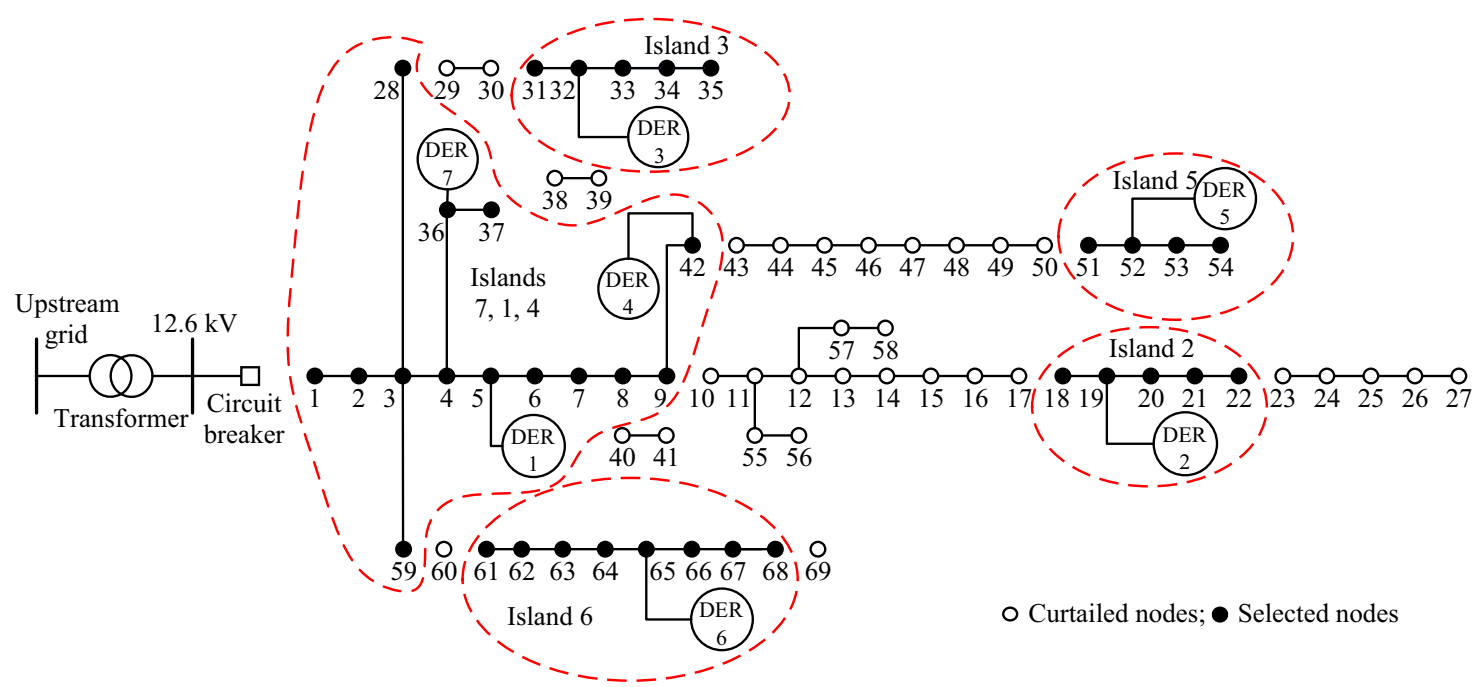

Fig. 7 Islanding formation by applying the proposed method in [17, 20]

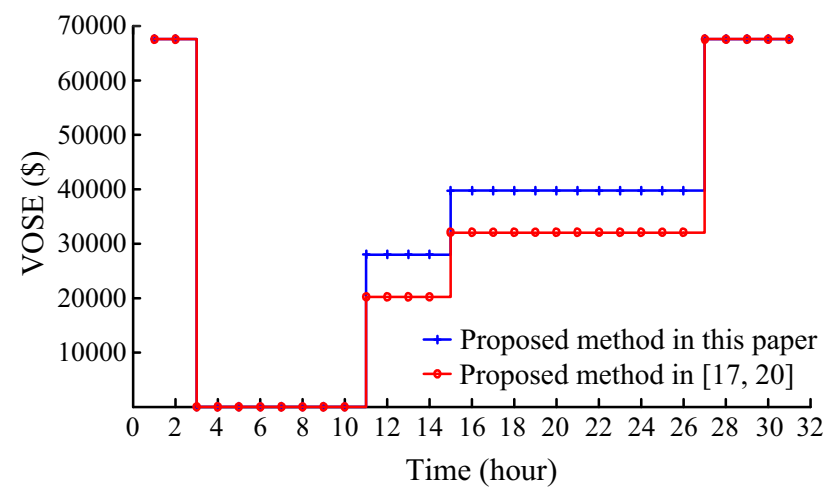

Fig. 8 Comparison of resilience of different islanding methods

It is assumed that the repair time for a substation is 24 hours, and gas turbine and the wind turbine can start operating 8 and 12 hours after the event, respectively. A fault occurs at the upstream substation at hour 3 , and it will be repaired at hour 27. Resilience curves, according to VOSE metric, is shown in Fig. 8 to compare the proposed method in this paper and the presented method in [17, 20]. The results show the improvement in the resilience by applying the proposed islanding method. In addition, the result shows that the PL index for the proposed method and the proposed method in $[17,20]$ are $21.5 \%$ and $27.5 \%$, respectively. Hence, PL is improved by more than $6 \%$ with the application of the proposed method. The reason for the improvement of resilience is the presence of a tie-switch between nodes 54 and 27.

It is worthwhile to investigate the impact of LPs on the islanding procedure. We design a case, in which the priority of all loads is assumed to be the same, equal to 1 . The results are presented in Fig. 9, and the details are reported in Table 6. Comparing Figs. 6 and 9, the selected optimal nodes are different in islands. For example, without priority, in island 2, DER 2 supplies nodes 15-20, where, with priority, DER 2 supplies nodes $18-22$ in island 2. Considering 1, 10, and 100 for the value of serving the classified demands, the VOSE in island 2 with LPs is 7155 $\$$ /hour which is $499.5 \$$ /hour more than that of the case without LPs. The results show that the total value of restored energy with and without LP is 39769 \$hour and 38891 \$/hour, respectively.

\subsection{Results of scenario 2}

In this scenario, simultaneous faults occur in the lines connecting nodes 3 and 59, nodes 17 and 18, and nodes 51 and 52, although the link between the network and the main grid remain connected. The results verify that in order to satisfy OPF constraints, the loads at nodes 24 and 69 should be shed by $4.2 \mathrm{~kW}$ and $14 \mathrm{~kW}$, respectively. The results shown in Fig. 10 depict that the distribution system is separated into two sections. One section remains connected to the upstream grid. DERs 1, 3, 4, 6, and 7 are also in this section for providing the demand. The other section operates in islanding mode, and DER 2 and DER 5 supply critical loads. In this case, the bus with the highest capacity DER, DER 5, is considered as slack bus and the buses with lower capacity DERs are considered as photovoltaic (PV) buses. The total amount of load curtailment and the VOSE would be $18.2 \mathrm{~kW}$ and $\$ 182.2$, respectively. In this case, DERs provide active power with maximum capacity and the tie-lines 1 and 3 transmit $114.2 \mathrm{~kW}$ and $86 \mathrm{~kW}$, respectively. 


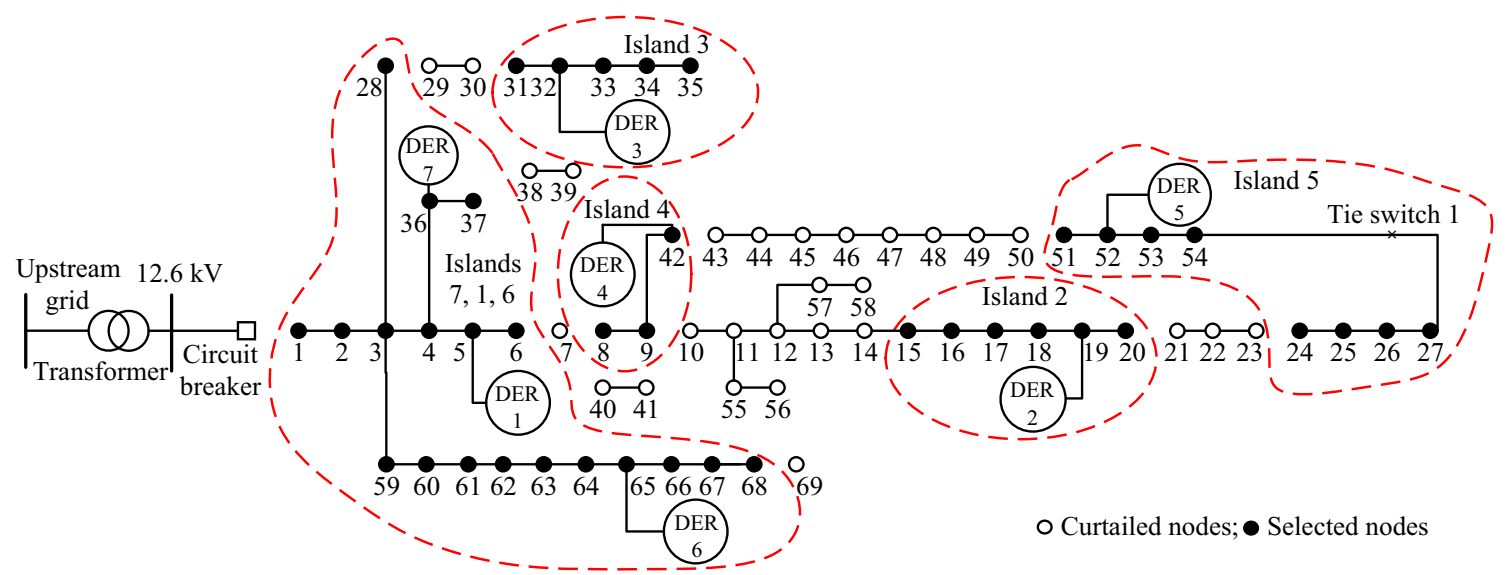

Fig. 9 Islanding formation by applying the proposed method in scenario 1 without LPs

Table 6 Characteristics of islands without LPs

\begin{tabular}{lllr}
\hline Island number & Candidate node & Selected node & Restored load (kW) \\
\hline 1 & $1-7,28,36,59$ & $1-6,28$ & 28.60 \\
2 & $13-27$ & $15-20$ & 182.50 \\
3 & $29-35$ & $31-35$ & 39.50 \\
4 & $8-10,43-47$ & $8,9,42$ & 109.35 \\
5 & $18-27,51-54$ & $24-27,51-54$ & 379.00 \\
6 & $1-6,14-15,28-36,59-69$ & $61-68$ & 94.40 \\
7 & Neutralized island 1, 4, 6, nodes 7,10, 29,36, & Neutralized islands 1, 6, nodes 36, & 157.00 \\
& $37,43-47,59,60,69$ & $37,59,60$ & \\
\hline
\end{tabular}

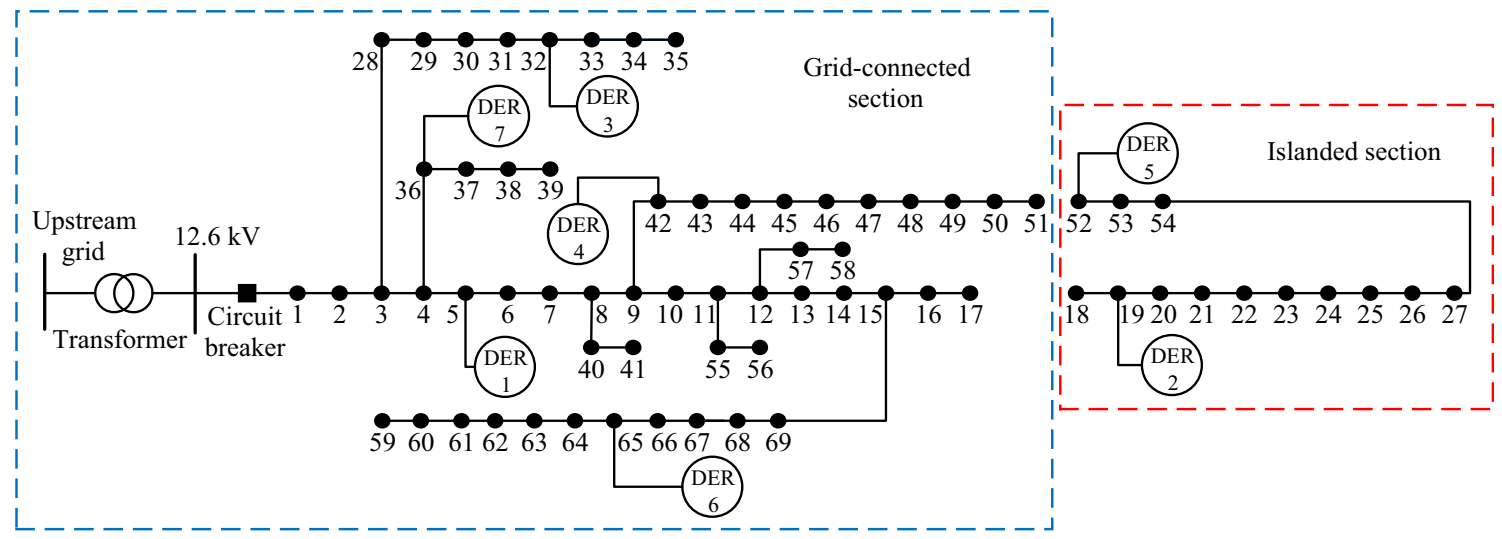

Fig. 10 Network reconfiguration by applying the proposed method in scenario 2 with LPs

It can be concluded from the case studies that:

1) In the case of disconnection between the distribution system and the upstream grid, intentional islanding is a more effective procedure for load restoration, as demonstrated by the results of scenario 1 .

2) Both intentional islanding and reconfiguration cooperate in the load restoration when the ADS is connected to the upstream grid. For example, in scenario 2, where the connection between nodes 3 and 59 is interrupted, tie-switch 3 is closed, and reconfiguration enhances the resilience of the network. However, their quota of restorationdepends on the technical constraints such as the line capacity of the tie-line.

3) Considering LP has an impact on the optimal island selection and the VOSE in the restoration procedure. 
Power quality [38], stability, and protection coordination [39] are important to be discussed to realize resilienceoriented intentional islanding of reconfigurable ADS. These topics may be interesting to be addressed in future works.

\section{Conclusion}

In this paper, a two-level intentional islanding method for a reconfigurable distribution system is proposed, considering the restoration of islanded loads by local DERs. To this end, in the first level, a TKP problem is modeled and solved. In the second level, the feasibility of the islands' operation is verified by solving an OPF problem. The results show that the operation of the primary islands can be optimized by controlling the reactive power generation of DERs and capacitor banks. In addition, the presence of tie-lines can improve the VOSE by $11.8 \%$ in comparison with the case without tie-lines. The application of the proposed method is also examined in the case of fault occurrences in different lines to show that the proposed method is valid in the resilience studies of the distribution systems. Considering the uncertainties of load and DER generation and severity of events is not in the scope of this paper. However, the authors are working on this issue, and the results will be reported upon accomplishment.

Open Access This article is distributed under the terms of the Creative Commons Attribution 4.0 International License (http:// creativecommons.org/licenses/by/4.0/), which permits unrestricted use, distribution, and reproduction in any medium, provided you give appropriate credit to the original author(s) and the source, provide a link to the Creative Commons license, and indicate if changes were made.

\section{References}

[1] Wang F, Chen C, Li C et al (2017) A multi-stage restoration method for medium-voltage distribution system with DGs. IEEE Trans Smart Grid 8(6):2627-2636

[2] Ding T, Lin Y, Bie Z et al (2017) A resilient microgrid formation strategy for load restoration considering master-slave distributed generators and topology reconfiguration. Appl Energy 199:205-216

[3] Arif A, Wang Z (2017) Networked microgrids for service restoration in resilient distribution systems. IET Gener Transm Distrib 11(14):3612-3619

[4] Marques LT, Delbem AC, London JB (2018) Service restoration with prioritization of customers and switches and determination of switching sequence. IEEE Trans Smart Grid 9(3):2359-2370

[5] El-Sharafy MZ, Farag HE (2017) Back-feed power restoration using distributed constraint optimization in smart distribution grids clustered into microgrids. Appl Energy 206:1102-1117

[6] Tsai MS (2008) Development of an object-oriented service restoration expert system with load variations. IEEE Trans Power Syst 23(1):219-225
[7] Kleinberg MR, Miu K, Chiang KHD (2011) Improving service restoration of power distribution systems through load curtailment of in-service customers. IEEE Trans Power Syst 26(3):1110-1117

[8] Chen C, Wang J, Qiu F et al (2016) Resilient distribution system by microgrids formation after natural disasters. IEEE Trans Smart Grid 7(2):958-966

[9] Chen C, Wang J, Ton D (2017) Modernizing distribution system restoration to achieve grid resiliency against extreme weather events: an integrated solution. Proc IEEE 105(7):1267-1288

[10] Wang Z, Wang J (2017) Service restoration based on AMI and networked MGs under extreme weather events. IET Gener Transm Distrib 11(2):401-408

[11] Weng JM, Liu D, Luo N et al (2015) Distributed processing based fault location, isolation, and service restoration method for active distribution network. J Mod Power Syst Clean Energy 3(4):494-503

[12] Gholami M, Moshtagh J, Ghadernejad N (2015) Service restoration in distribution networks using combination of two heuristic methods considering load shedding. J Mod Power Syst Clean Energy 3(4):556-564

[13] Hong HF, Hu ZS, Guo RP et al (2017) Directed graph-based distribution network reconfiguration for operation mode adjustment and service restoration considering distributed generation. J Mod Power Syst Clean Energy 5(1):142-149

[14] Basso TS, DeBlasio R (2004) IEEE 1547 series of standards: interconnection issues. IEEE Trans Power Electron 19(5):1159-1162

[15] Quevedo PM, Contreras J, Rider MJ et al (2015) Contingency assessment and network reconfiguration in distribution grids including wind power and energy storage. IEEE Trans Sustain Energy 6(4):1524-1533

[16] Liang J, Wang X, Wang P et al (2012) Two-stage method for optimal island partition of distribution system with distributed generations. IET Gener Transm Distrib 6(3):218-225

[17] Oboudi MH, Hooshmand R, Karamad A (2016) Feasible method for making controlled intentional islanding of microgrids based on the modified shuffled frog leap algorithm. Int $\mathbf{J}$ Electr Power Energy Syst 78:745-754

[18] Oboudi MH, Hooshmand R, Karamad A (2017) A feasible method for controlled intentional islanding in microgrids based on PSO algorithm. Swarm Evolut Comput 35:14-25

[19] Hosseinnezhad V, Rafiee M, Ahmadian M et al (2018) Optimal island partitioning of smart distribution systems to improve system restoration under emergency conditions. Int J Electr Power Energy Syst 97:155-164

[20] Zhang M, Chen J (2015) Islanding and scheduling of power distribution systems with distributed generation. IEEE Trans Power Syst 30(6):3120-3129

[21] Arefifar SA, Mohamed YARI (2014) DG mix, reactive sources and energy storage units for optimizing microgrid reliability and supply security. IEEE Trans Smart Grid 5(4):1835-1844

[22] Awad AS, El-Fouly TH, Salama MM (2014) Optimal ESS allocation and load shedding for improving distribution system reliability. IEEE Trans Smart Grid 5(5):2339-2349

[23] El-Zonkoly A, Saad M, Khalil R (2013) New algorithm based on CLPSO for controlled islanding of distribution systems. Int J Electr Power Energy Syst 45(1):391-403

[24] El-Zonkoly A (2017) Optimal scheduling of observable controlled islands in presence of energy hubs. Electr Power Syst Res 142:141-152

[25] Hussain A, Bui VH, Kim HM (2017) Optimal operation of hybrid microgrids for enhancing resiliency considering feasible islanding and survivability. IET Renew Power Gener 11(6):846-857 
[26] Li ZY, Shahidehpour M, Aminifar F et al (2017) Networked microgrids for enhancing the power system resilience. Proc IEEE 105(7):1289-1310

[27] Stankovic A (2018) The definition and quantification of resilience. https://resourcecenter.ieee-pes.org/technical-publications/ technical-reports/PESTR0065_04-18.html. Accessed 6 April 2018

[28] Panteli M, Mancarella P (2017) Modeling and evaluating the resilience of critical electrical power infrastructure to extreme weather events. IEEE Syst J 11(3):1733-1742

[29] Ding T, Sun K, Huang K et al (2018) Mixed-integer linear programming-based splitting strategies for power system islanding operation considering network connectivity. IEEE Syst J 12(1):350-359

[30] Mandelli S, Brivio C, Colombo E et al (2016) A sizing methodology based on levelized cost of supplied and lost energy for off-grid rural electrification systems. Renew Energy $89: 475-488$

[31] Sullivan MJ, Mercurio M, Schellenberg J (2009) Estimated value of service reliability for electric utility customers in the United States (No. LBNL-2132E). Lawrence Berkeley National Laboratory (LBNL), Berkeley

[32] Even S (2011) Graph algorithms. Cambridge University Press, Cambridge

[33] Albadi MH, El-Saadany EF (2007) Demand response in electricity markets: an overview. In: Proceedings of IEEE PES general meeting, Tampa, USA, 24-28 June 2007, 5 pp

[34] Gonzalez-Longatt FM, Rueda JL (2014) Power factory applications for power system analysis. Springer, Berlin

[35] Savier JS, Das D (2007) Impact of network reconfiguration on loss allocation of radial distribution systems. IEEE Trans Power Deliv 22(4):2473-2480

[36] One H (2009) Distributed generation technical interconnection requirements-interconnection at voltages $50 \mathrm{kV}$ and below. Report, Toronto

[37] Atwa YM, El-Saadany EF (2009) Reliability evaluation for distribution system with renewable distributed generation during islanded mode of operation. IEEE Trans Power Syst 24(2):572-581
[38] Gayatri MTL, Parimi AM, Kumar AP (2016) Utilization of unified power quality conditioner for voltage sag/swell mitigation in microgrid. In: Proceedings of 2016 biennial international conference on power and energy systems: towards sustainable energy (PESTSE), Bengaluru, India, 21-23 January 2016, 6 pp

[39] Zhao Z, Yang P, Guerrero JM et al (2015) Multiple-time-scales hierarchical frequency stability control strategy of mediumvoltage isolated microgrid. IEEE Trans Power Electron 31(8):5974-5991

Mohammad Hossein OBOUDI received the M.Sc. degree from the University of Isfahan, Isfahan, Iran, in 2013. He is currently pursuing the Ph.D. degree in the School of Electrical and Computer Engineering of Shiraz University, Shiraz, Iran. His research interests include power system resiliency and reliability studies, microgrids and renewable energy operation.

Mohammad MOHAMMADI received the B.Sc. degree from Shiraz University, Shiraz, Iran, in 2000, and the M.Sc. and Ph.D. degrees from the Amirkabir University of Technology, Tehran, Iran, in 2002 and 2008, respectively. He is currently an associate professor with the Department of Power and Control Engineering, Shiraz University, Shiraz, Iran. His research interests include power system probabilistic analysis, power system security assessment, machine learning, and power system dynamic analysis.

Mohammad RASTEGAR received the B.Sc., M.Sc., and Ph.D. degrees from the Sharif University of Technology, Tehran, Iran, in 2009, 2011, and 2015, respectively, all in electrical engineering. He joined the School of Electrical and Computer Engineering, Shiraz University, Shiraz, Iran, in 2016. His research interests include modeling home energy management systems, plug-in hybrid electric vehicle operation, and power system reliability and resiliency studies. 